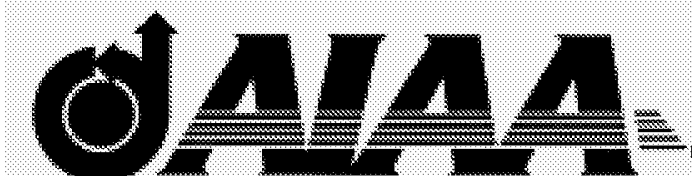

AIAA 2002-2555

Experiments and Analyses of

Distributed Exhaust Nozzles

K.W. Kinzie

NASA Langley Research Center

D.B. Schein and W.D. Solomon, Jr.

Northrop Grumman Corporation

$8^{\text {th }}$ AIAA/CEAS Aeroacoustic Conference

17-19 June 2002

The Great Divide Lodge

Breckenridge, Colorado

For permission to copy or to republish, contact the copyright owner named on the first page.

For AIAA-held copyright, write to AIAA Permissions Department,

1801 Alexander Bell Drive, Suite 500, Reston, VA 20191-4344 


\title{
EXPERIMENTS AND ANALYSES OF DISTRIBUTED EXHAUST NOZZLES
}

\author{
Kevin W. Kinzie ${ }^{*}$ \\ NASA Langley Research Center, MS 166 \\ Hampton, VA 23681 \\ David B. Schein and W. David Solomon, Jr. ${ }^{* *}$ \\ Northrop Grumman Integrated Systems \\ El Segundo, CA 90245-2804
}

\begin{abstract}
Experimental and analytical aeroacoustic properties of several distributed exhaust nozzle (DEN) designs are presented. Significant differences between the designs are observed and correlated back to CFD flowfield predictions. Up to $20 \mathrm{~dB}$ of noise reduction on a spectral basis and $10 \mathrm{~dB}$ on an overall sound pressure level basis are demonstrated from the DEN designs compared to a round reference nozzle. The most successful DEN designs acoustically show a predicted thrust loss of approximately $10 \%$ compared to the reference nozzle. Characteristics of the individual mini-jet nozzles that comprise the DEN such as jet-jet shielding and coalescence are shown to play a major role in the noise signature.
\end{abstract}

\section{Introduction}

Jet noise continues to be a dominant aircraft noise source that limits operations of current aircraft and hinders the design of future aircraft. While techniques aimed at changing the engine cycle or those implementing mixing enhancement devices are incrementally improving the community noise situation, revolutionary improvements in conventional engine/airframe systems are required to meet NASA's perceived noise reduction goal of $20 \mathrm{~dB}$ in 25 years. One such concept with potential to make significant progress toward the 25-year goal is the distributed exhaust nozzle (DEN).

Noise suppression from the DEN concept results from a favorable shift in the spectral shape of the radiated jet noise. The smaller jets that comprise the distributed exhaust nozzle radiate noise at higher frequencies than larger single or dual flow exhaust nozzles. Atmospheric attenuation increases nearly exponentially with increasing frequency, and spectral noise components contribute less and less to the EPNL noise metric as the frequency increases above $4 \mathrm{kHz}$. In fact, noise at frequencies higher than $10 \mathrm{kHz}$ is not even included in the calculation of EPNL. In addition to shifting the noise signature toward more favorable high frequencies, the small jets mix with the ambient air and reduce the speed and temperature of the jet plume to lower levels that, in turn, reduce the radiated low frequency noise.

Traditionally, distributed exhaust nozzle concepts have been studied from the perspective of replacing conventional engine exhaust nozzles with another configuration composed of many small tubes, chutes, or spokes. However, this inevitably leads to high levels of base drag due to the aft facing area required to distribute the exhaust. NASA Langley Research Center is conducting research aimed at studying the distributed exhaust concept from an integrated exhaust/airframe system perspective where the propulsion system is integrated into the airframe and the small exhaust nozzles are distributed over large portions of the wing surface area. An integrated distributed exhaust propulsion system has further potential for noise reduction since additional noise suppression will be realized through shielding of engine noise away from the community by the airframe design.

In 2000, NASA teamed with Northrop Grumman Corporation (NGC) to design and test a horizontal slot nozzle ${ }^{1}$ concept in the Low Speed Aeroacoustic Wind Tunnel (LSAWT), shown in Figure 1. While the LSAWT DEN configuration provided only minimal noise suppression, the agreement between computational fluid dynamics (CFD) predictions andhrust and flow field measurements was sufficient to justify continued application of NGC's CFD design approach in pursuit of more aggressive noise reduction. CFD has subsequently been used to

\footnotetext{
* Senior Member, AIAA

** Member, AIAA
} 
design three new DEN nozzles for testing in the LaRC Small Anechoic Jet Facility (SAJF).

Two of the designs reported here are variations of ones considered for the previous LSAWT test ${ }^{1}$. Due to the cost and complexity of fabrication, the horizontal slot design was chosen for that test even though it had less potential for noise reduction than other designs. In order to investigate more aggressive acoustic designs, a simpler approach was chosen for the current test by fabricating the DEN models using a stereolithography process. While this restricts the flow temperature to less than $150^{\circ} \mathrm{F}$, it provides a means of inexpensively screening designs.

The third design was a smaller version of the horizontal slot design ${ }^{1}$. Even though the horizontal slot design does not provide significant noise reduction, testing the smaller stereolithography model provided a link back to the larger scale, hot-flow model test. While the SAJF horizontal slot data are not reported here, the acoustic characteristics including spectral content and relative levels compared to the round reference nozzle were similar to those found in the larger LSAWT design. This gives confidence that the trends and observations of the two new DEN designs can also be expected as the model size increases and hot flow is used.

\section{Nozzle Descriptions}

For this work, the two new SAJF DEN designs were analyzed, tested and compared to a round reference nozzle with similar mass flow. The DEN designs were refined using CFD in an attempt to optimize aero-performance and mixing characteristics that affect noise radiation. As expected, compromises are required between these two generally opposing requirements. The first DEN design is shown in Figure 2 and will be referred to as the DROPS design because the exit holes are shaped like teardrops. Figure 3 shows the second DEN design and will be referred to as the slanted pseudo-slot (SPS) design. It is similar to the design of the slotted nozzle, also designed by Northrop Grumman, described in Gaeta, et $\mathrm{al}^{2,3}$ except that for the current design the exit passages are not continuous slots, but rather an array of rectangular nozzles that give the appearance of slots. In addition, the spanwise spacing of the rows for the current SPS design is further apart than that tested by Gaeta, et $\mathrm{al}^{2,3}$. For the current design, the spacing is 5.5 times the slot width and for the Gaeta, et al. ${ }^{2,3}$ design the spacing is 3.5 times the slot width. The round reference nozzle was constructed of stainless steel while the two DEN designs were constructed using a stereolithography technique.

Figure 4 shows a sketch describing the coordinate system used for this work. An azimuthal angle of $\phi=0^{\circ}$ is the horizontal plane of symmetry as the nozzles are shown and an azimuthal angle of $\phi=90^{\circ}$ is the vertical plan of symmetry as shown. Therefore, for a measurement angle of $\phi=0^{\circ}$, the furthest row of mini-nozzles is shielded from the microphones by the closer rows. For a measurement angle of $\phi=90^{\circ}$, the microphones are directed at all the mini-nozzle rows equally. Polar directivity angles are designated by $\theta$ and are measured from the jet inlet axis with $\theta=180^{\circ}$ being aligned directly on the jet axis in the downstream direction.

The round reference nozzle had a 2 -inch diameter for a total exit area of $3.14 \mathrm{in}^{2}$. Several CFD iterations were performed to size the distributed exhaust nozzles such that they had approximately the same mass flow as the round nozzle. This resulted in the SPS design having an exit area of $3.32 \mathrm{in}^{2}$ and the DROPS design having an exit area of $3.25 \mathrm{in}^{2}$. The individual mininozzles for the SPS design each had an area of approximately $0.030 \mathrm{in}^{2}$ and a single mini-nozzle for the DROPS design had an area of approximately $0.032 \mathrm{in}^{2}$.

\section{Experimental Approach}

The nozzles were tested in NASA Langley's Small Anechoic Jet Facility (SAJF). An eight-element microphone array on an approximate 7 foot sideline was used to measure radiated noise. Because of the size of the chamber and of the nozzles, acoustic measurements were only made in the aft quadrant from polar angles of $\theta=90^{\circ}$ to $\theta=155^{\circ}$. Narrowband data up to $100 \mathrm{kHz}$ were acquired with $1 / 4$ " B\&K 4139 microphones and post-processed to standard $1 / 3$ octave bands. The data were then extrapolated to a 12 foot arc centered at the nozzle exit and corrected to standard day reference conditions using the Shields and Bass atmospheric attenuation model ${ }^{4}$

A range of nozzle pressure ratio (NPR) conditions were measured starting from NPR $=1.45$ and ending with $\mathrm{NPR}=2.20$. For all pressure conditions, the flow total temperature was held at $120^{\circ} \mathrm{F}$. The flow temperature was limited to relatively cool conditions by the plastic stereolithography models. This paper focuses on the NPR $=1.72$ condition since most of the CFD was run for this case, which also corresponds to the fully mixed take-off pressure ratio for the cycle conditions tested previously for the horizontal slot DEN design'. Four azimuthal planes were measured corresponding to $\phi=0^{\circ}, 30^{\circ}, 60^{\circ}$, and $90^{\circ}$. Only the $\phi=0^{\circ}$ and $\phi=90^{\circ}$ planes are reported in this paper as the intermediate angles did not provide significant additional insight. 


\section{CFD Analysis}

CFD simulations were run on the reference nozzle and both DEN designs using Northrop Grumman's Generalized Compressible Navier-Stokes (GCNS) $\operatorname{code}^{5}$. Full quadrant solutions were obtained for the reference nozzle and the SPS design. The DROPS design was analyzed using 3D strip analysis with periodic boundary conditions due to time constraints. The CFD was performed at nozzle pressure ratios of $1.45,1.72$, and 2.20. However, the most detailed analysis was performed at NPR $=1.72$. The total temperature for all CFD runs was $120^{\circ} \mathrm{F}$.

Figure 5 shows predicted velocity contours through the hole spanwise centers for the reference nozzle and the DEN designs. The DEN designs show a dramatic increase in mixing that results in a plume with significantly lower flow velocity than the round reference nozzle. This is in contrast to the horizontal slot DEN design ${ }^{1}$ that showed very little reduction in plume velocity compared to the reference nozzle. Figure 6 shows predicted turbulence intensity contours for the same cross sections. Again, there is a significant reduction in turbulence intensity for the DEN designs compared to the reference nozzle.

The CFD predictions show that for both of these DEN designs, the individual mini-exhaust jets maintain their identity for a significant distance before they begin to coalesce back into a larger jet plume. This is a critical factor in order for acoustic suppression to be realized from any distributed exhaust design. CFD predictions and experimental measurements of the horizontal slot DEN design ${ }^{1}$ showed that without enough separation of the mini-jets, they will coalesce into a large plume with a noise signature more characteristic of the single large jet rather than many small jets. The CFD flowfield solutions for the current DEN designs show much greater potential for noise reduction based on jet-to-jet mixing and overall plume characteristics. The acoustic measurements presented in the next section confirm this assessment.

For the design nozzle pressure ratio of 1.72 , the discharge coefficient of the SPS nozzle was 0.93 while the discharge coefficient of the DROPS nozzle was 0.95. The thrust coefficient of both the SPS and DROPS nozzles was approximately 0.89 . The CFD showed a slight improvement in thrust performance (0.91) as the NPR increased to 2.20. In addition, preliminary computations also indicate a performance increase at forward flight speeds. Experimental measurements of aero-performance quantities were not made, however, earlier work demonstrated that the GCNS code is very reliable in computing these types of flows ${ }^{1}$.
Caution should be used when comparing acoustic data from nozzles with different thrust characteristics. An aircraft system requires a specific level of thrust so a nozzle with lower thrust must either be oversized or operated at a higher pressure ratio to generate the same amount of thrust as one with better propulsive efficiency. Either of these options will increase the noise so it can be misleading to only consider noise suppression without considering the associated performance penalty. As a point of future reference, oversizing the DEN designs discussed here so that they would produce the same thrust as the round reference nozzle would result in an elevation of the DEN SPL levels shown in this paper by approximately $0.5 \mathrm{~dB}$.

While the thrust loss of these DEN specimens may be unacceptable for conventional aircraft designs, the distributed exhaust concept lends itself very well toward more revolutionary aircraft designs where the small exhaust holes can be integrated into the wing surface. Such an installation could recover some of the lost performance through upper surface blowing lift enhancement. A full aircraft system mission study is required to adequately assess the acceptable thrust loss compared to the noise suppression provided by the distributed exhaust design.

\section{Acoustic Measurements}

Figures 7 and 8 show overall sound pressure level (OASPL) directivity data for the round reference nozzle compared to the DROPS and SPS nozzles at a nozzle pressure ratio of 1.72 . Azimuthal variations of $\phi=0^{\circ}$ and $\phi=90^{\circ}$ are shown for the DEN designs. Both nozzles provide greatest noise suppression at the aft most angles where jet noise is typically the loudest. The $\phi=0^{\circ}$ orientation of the DROPS nozzle is nearly $10 \mathrm{~dB}$ quieter than the round reference nozzle at the aft most measurement angles. The DROPS nozzle shows a consistent noise benefit relative to the reference nozzle through the whole measurement range. The SPS nozzle does not achieve suppression until midway into the aft quadrant. For each DEN, there is a loud and a quiet azimuthal orientation with the $\phi=0^{\circ}$ orientation being 2 $-3 \mathrm{~dB}$ quieter than $\phi=90^{\circ}$. Effects that contribute to the difference between azimuthal planes will be discussed shortly.

Spectra from the two DEN designs compared to the round reference nozzle at NPR $=1.72$ are shown in Figures 9 through 12. Figures 9 and 10 show the DROPS and SPS for both azimuthal angles close to the peak noise polar angle of $\theta=150^{\circ}$. Figures 11 and 12 show the DROPS and SPS, respectively, for both azimuthal angles and a polar angle of $\theta=90^{\circ}$. From these spectra, it is clear that the DEN models are in fact 3

American Institute of Aeronautics and Astronautics 
shifting the noise to higher frequencies as designed. Significant noise reduction up to $20 \mathrm{~dB}$ is observed for some frequency bands compared to the round reference nozzle, which has a noise peak an order of magnitude lower in frequency than the DEN models. Consistent with the OASPL plots, the DROPS nozzle provides more noise reduction than the SPS nozzle. It is hypothesized that the DROPS nozzle provides greater separation between the mini-jets and provides more overall mixing that results in greater noise reduction.

Note that at an azimuthal orientation of $\phi=90^{\circ}$, particularly at a polar angle of $\theta=90^{\circ}$, there is a low frequency local maximum in the DEN spectrum that does not occur in the $\phi=0^{\circ}$ azimuthal orientation. Both DEN designs show a low frequency peak around $1000 \mathrm{~Hz}$. This characteristic spectral "hump" is also observed in the slanted slot DEN reported in Gaeta, et $\mathrm{al}^{2,3}$ in the same frequency range found here.

The distinct azimuthal directivity pattern of the $1000 \mathrm{~Hz}$ noise hump is characteristic of a dipole source with a $\cos (\phi)$ directivity pattern. The similarity of the hump between the SPS, DROPS, and slanted slot design also implies that a common design characteristic may be causing this noise. Gaeta, et $\mathrm{a}^{2}$ present strong evidence tying this noise to a turbulent boundary layer - trailing edge source related to the nozzles' closeout geometry. This hypothesis could be confirmed by altering the trailing edge in a way that would alter the source coherence. However, such modifications were not made during this test series. Additional analysis of the data collected from the SPS and DROPS nozzles will be performed using to establish that velocity scaling $\left(V^{5}\right)$ and directivity pattern characteristics are typical of a trailing edge noise source. Noise source prediction tools $^{7}$ used to isolate airfoil noise sources during analysis of the earlier horizontal slot nozzle data ${ }^{l}$ will be applied, to further investigate the source of the $1000 \mathrm{~Hz}$ noise peak.

It is highly likely that this noise source is characteristic of these specific designs and could be removed in future designs or in integrated configurations. The resultant acoustic characteristics would thus be even more attractive than what has been shown here. Based on comparing the spectra from the two azimuthal planes in Figures 9 through 12, this hump is the primary factor in the 2 to $3 \mathrm{~dB}$ OASPL difference between the two azimuthal planes shown in Figures 7 and 8, especially toward the sideline polar angles. Therefore, reducing the trailing edge noise source would lower the $\phi=90^{\circ}$ azimuthal plane noise to nearly what is measured in the quieter $\phi=0^{\circ}$ azimuthal plane.

Figures 13 and 14 show plots of the noise difference between the DROPS and SPS nozzles and the reference nozzle at three different pressure ratios for azimuthal angle $\phi=0^{\circ}$ and polar angle of $\theta=150^{\circ}$. Negative SPL values represent noise reduction while positive values indicate frequency bands louder than the round reference nozzle. Again, it is observed that the DROPS nozzle typically provides more noise reduction than the SPS nozzle. Not only does the DROPS nozzle provide more noise reduction at low frequencies, it generates less noise at high frequencies. Both nozzles provide the greatest noise reduction in the frequency range of 1 to $2 \mathrm{kHz}$, with increased noise reduction as the NPR increases. At high frequencies where excess noise is generated compared to the round reference nozzle, the two DEN designs show opposite trends. For the DROPS design, more noise is generated as NPR increases. For the SPS design, less excess noise is generated as NPR increases.

It is worthy to note that all of the acoustic trends observed here are generally consistent with those for the slanted slot DEN reported by Gaeta, et $\mathrm{al}^{2,3}$. One notable difference is that the SPS nozzle reported here provides more noise reduction than the first generation slanted slot nozzle, especially at subsonic pressure ratios. The enhanced noise reduction is likely due to the previously mentioned increased spanwise spacing between the slots which allows better mixing characteristics for the SPS design compared to the slotted design tested by Gaeta, et $\mathrm{al}^{2,3}$.

In contrast to the horizontal slot DEN design tested previously ${ }^{1}$, both current designs provide significant noise reduction. As mentioned earlier, it is clear from the CFD that the current designs achieve much better mixing by maintaining the individual mini-jets for a longer distance before they coalesce back into a larger plume. This separation is very important in achieving the low frequency noise reduction observed here and to shift the peak acoustic frequency to higher values. However, we also note that there is an aeroperformance penalty associated with achieving the jetjet separation and enhanced noise reduction. One of the long-term goals for DEN research is to minimize this penalty while improving or maintaining noise reduction.

Pre-test expectations for DEN acoustic performance have for earlier efforts been based on qualitative assessments of graphical CFD predictions. These assessments have been based on an understanding of Lighthill's jet noise analogy, relying on visual comparisons of velocity, turbulence intensity (like those shown in Figures 5 and 6), and turbulence length scale contours to those for the reference nozzle. Ideally, a computational aeroacoustics (CAA) model would be used in combination with CFD to predict the jet noise from new designs. Since this capability is not yet in place, a first order, non-rigorous, estimating method was derived from Lighthill's equation for jet 
noise and dimensional analysis. The development of this "noise parameter" is documented in the SAJF model design report ${ }^{5}$. Lighthill's equation for far-field acoustic pressure perturbation (above ambient) in the absence of surfaces is, in time derivative form:

$$
\bar{p}(\underline{x}, t)-p_{\infty}=\frac{x i x_{j}}{4 \pi c_{\infty}^{2}|\underline{x}-\underline{y}|_{V}^{3}} \int_{V}\left[\frac{\partial^{2} \overline{T_{i j}}(\underline{y}, \tau)}{a^{2}}\right] d \underline{y}
$$

where $\overline{T_{i j}}=\rho \overline{\mathrm{U}_{\mathrm{i}} \mathrm{U}_{\mathrm{j}}} \quad$ is the approximate applied stress field, ignoring viscous stresses and compressibility effects, $\underline{v}$ is the distance from a reference point (e.g. the nozzle exit) to the source point, $x$ is the distance to the far field observation point (the $x_{\mathrm{i}}$ representing its components), $c_{\infty}$ is the ambient sound speed, and $\tau$ is the retarded time. Integration is over all significant source volume. Writing $\mathrm{U}_{k}=U_{k}+u_{\mathrm{k}}^{\prime}$, the sum of a mean and fluctuation, respectively, we have $\mathrm{U}_{i} \mathrm{U}_{j}=U_{i} U_{j}+U_{i} u_{j}^{\prime}+U_{j} u_{i}^{\prime}+u_{i}^{\prime} u_{j}^{\prime}$. The first term is independent of time, and the fourth term is of higher order than the second and third terms, which are symmetric, so we have $\overline{T_{i j}}=2 \rho \overline{U_{j} u_{i}}$. Assuming constant density, the equation for acoustic pressure $p^{\prime}(\underline{x}, t)=\bar{p}(\underline{x}, t)-p_{\infty}$ is now:

$p^{\prime}(\underline{x}, t)=\frac{\rho x_{i} x_{j}}{2 \pi c_{\infty}^{2}|\underline{x}-\underline{y}|^{3}} \int_{V} \frac{\partial^{2}}{\partial t^{2}}\left[U_{j}(\underline{y}) u_{i}^{\prime}\left(\underline{y}, t-\frac{x}{c_{\infty}}\right)\right] d \underline{y}$

To first order, dimensional analysis ${ }^{8}$ gives $\partial^{2} / a^{2} \sim 1 / t_{\text {char }} \sim u^{\prime 2} / L^{2}$ where $\mathrm{t}_{\text {char }}$ is the characteristic time, taken as the turbulent eddy turnover time, $u^{\prime}$ is the velocity fluctuation, and $\mathrm{L}$ is the turbulence length scale. Note that $U_{j}$ is dominated by the component in the streamwise direction, and $\mathrm{u}_{i}^{\prime}$ is of the same order for each direction, so that $u_{1}^{\prime 2} \approx u_{2}^{\prime 2} \approx u_{3}^{\prime 2}$. A simplified expression for use in qualitative comparison of the RMS acoustic pressure pressure squared for each set of CFD flow fields is then:

$$
p^{\prime 2} \sim \frac{\rho^{2} V^{2}}{4 \pi^{2} c_{\infty}^{4} L^{4} r^{2}}\left[u_{1}^{\prime 4}(\underline{y}) U_{1}^{2}(\underline{y}) u_{1}^{\prime 2}(\underline{y})\right]
$$

On a decibel scale, Equation (3) can be written,

$$
10 \log _{10}\left(p^{\prime 2}\right) \sim K+10 \log _{10}\left[u_{1}^{\prime 6}(\underline{y}) U_{1}^{2}(\underline{y})\right]
$$

For the purpose of this first order quantitative comparison, we refer to the second term on the right side of Equation 4, $10 \log _{10}\left(\mathrm{u}_{1}^{\prime 6} \mathrm{U}_{1}^{2}\right)$, as the "noise parameter." This expression (which considers the axial component of turbulence only) omits factors representing effects of the turbulence length scale and source volume.

The noise parameter was computed throughout the $\mathrm{NPR}=1.72$ flow fields using CFD output for both current and earlier DEN designs. To provide a foundation for the usefulness of these calculations, CFD solutions obtained for the previously tested horizontal slot nozzle ${ }^{1}$ and its $2 \mathrm{D}$ reference nozzle were used to compute their respective noise parameter fields, also for $\mathrm{NPR}=1.72$. The noise parameter contour results are shown in Figure 15. Each picture represents a section cut through the nozzle's spanwise center. The parameter is normalized by the jet exit velocity, $U_{j}$, of the respective reference nozzle. Diagrams $A$ and $B$ show calculations for the previously tested $2 \mathrm{D}$ reference and horizontal slot configurations, respectively. Note that there is not a significant difference between the characteristics of the two fields, except that the "loudest" area (red / orange contour) extends somewhat farther downstream in the case of the $2 \mathrm{D}$ reference. This observation is consistent with measured data from the LSAWT test that, as mentioned earlier, produced only minimal noise reduction at low frequencies from the horizontal slots. Diagrams C, D, and E show simulation results for the subject round reference nozzle, the DROPS DEN geometry, and the SPS DEN geometry, respectively. For these cases there is a dramatic reduction in the noise parameter for each of the distributed nozzles, with the largest values in the downstream DEN plumes reduced by about $15 \mathrm{~dB}$ from those of the reference. This value appears reasonable considering the data shown earlier.

However, caution must be used in making predictions of noise performance using this simple parameter for a number of reasons, including: a) the parameter includes only axial turbulence fluctuations and is therefore most valid for noise radiation at $\theta=0^{\circ}$ and $\theta=180^{\circ}$, b) calculations are made point-by-point, with no source-volume integration, and c) turbulence length scale and volume are omitted, but could be included as a separate factor. Despite these simplifications, the noise parameter defined here and illustrated in Figure 15 appears to provide a first-order indication of DEN acoustic performance relative to a conventional reference nozzle.

\section{Jet-Jet Noise Shielding Studv}

Characteristic to the DEN technology is a significant reduction in low frequency noise accompanied by an increase in the high frequency

5

American Institute of Aeronautics and Astronautics 
components of noise. For these size nozzles, the crossover point between noise suppression and the high frequency excess noise generated compared to the round reference ranges between $6 \mathrm{kHz}$ and $10 \mathrm{kHz}$. The peak frequency for both DEN designs is in the range of $15 \mathrm{kHz}$. This crossover point is problematic when it comes to projecting the scale model DEN to full size. Typically, Strouhal number scaling using a scale factor related to the nozzle exit area is applied that shifts model scale frequency in proportion to the geometric scale factor. If a single factor is used to scale the model-scale DEN acoustic spectra then the high frequency cross-over would cause the DEN models to be much louder than the round reference nozzle and these frequencies would dominate the sound field on a perceived noise level (PNL) basis. However, the model scale DEN designs have two length scales. One is associated with the mini-nozzles, which are close to the actual size they would be full scale, and the other is associated with the overall nozzle exit area which is much smaller than what it would be full size. Consequently, the lower frequency energy that is generated by the coalesced jet plume should scale to even lower frequency, but the higher frequency energy generated by the individual mini-jets would not shift frequency. In addition, jet-jet acoustic shielding by the arrays of nozzles will be a significant noise reduction effect that may increase with DEN model size. Thus, a full-scale DEN may provide even more suppression on a PNL basis than observed here.

The acoustic shielding effect has been investigated thoroughly in several twin-jet studies ${ }^{9,10,11}$, but only recently for DEN designs ${ }^{2,3}$. Our objective here was to obtain nozzle array acoustic shielding data that might aid in scaling at least part of the DEN acoustic spectrum for larger (greater flow area) nozzle systems. It is important to note that the DEN models tested here are considered to be small, full-scale sections of a larger nozzle system (i.e. the mini-nozzles in a full size system will be the same size as tested here, there will just be a lot more of them).

To quantify the shielding effect for the current SPS design, data were acquired from modified configurations realized by successively blocking (internally) neighboring slots on both the top and bottom array. First slot \#7 was blocked and data acquired, then $\# 6$ and $\# 7$ were blocked, etc. until only the \#1 slot was flowing. For each of these test points, mass flow was adjusted to maintain the desired value of NPR. In this way, the acoustic effect of adding each individual subsequent slot flow could be measured. The nozzle's azimuthal orientation was $\phi=0^{\circ}$ for all shielding runs. Data obtained for $\mathrm{NPR}=1.72$ are presented here in Figures 16 through 18.
Measured one-third-octave band spectra for the $\theta=150^{\circ}$ directivity angle are shown in Figure 16 . For all bands above about $2000 \mathrm{~Hz}$ the spread between spectra is less than $4 \mathrm{~dB}$, and data for the two slots through six slots cases are nearly indistinguishable demonstrating that once two of the slots are flowing, adding additional slot flow did not appreciably increase the noise in the high frequency range. The slight increase when the seventh slot is open is likely due to model edge entrainment. The larger spread in the low frequency data is an indication of downstream coalescence of the individual exhaust plumes generating excess noise. However, this appears to occur primarily in moving from one to two slots.

Using linear acoustic theory, the change in SPL obtained by operating fewer than seven slots can be estimated. The noise reduction relative to the sevenslot case expected from $N$ flowing slots, independent of mixing and shielding effects, is given by $10 \log (N / 7)$. This quantity is calculated for each test case in Table 1. As an example, if only 1 slot is flowing with no interaction effects between the slots, Table 1 shows that the resulting SPL would be approximately $8.45 \mathrm{~dB}$ lower than if all seven slots were flowing.

To more easily evaluate the effects of jet shielding and plume coalescence on the measured spectra, data for each test point were normalized as follows. The appropriate value from Table 1 was added to each spectrum shown in Figure 16, to correct SPL levels for the number of flowing slots. Next, the spectrum levels measured for all seven slots flowing was subtracted from each of these spectra. Therefore, for a system of completely independent jets, these normalizations would result in $0 \mathrm{~dB}$ spectra at all frequencies. The spectra generated in this way corresponding to those of Figure 16 are shown in Figure 17. For a given data point, net negative SPL spectral values represent frequencies for which shielding of jet noise by neighboring slots occurs. Net positive SPL values indicate frequencies for which merging of minijets results in excess mixing noise. Data presented in Figure 17 show that for $\theta=150^{\circ}$, there is a large acoustic shielding effect for mid to high frequencies, while at low frequencies, downstream coalescence of the mini-plumes generates extra low frequency noise. As noted above, most of this appears to occur in going from the one-slot to two-slot geometry.

Normalized data for the NPR $=1.72, \theta=90^{\circ}$ measurements are shown in Figure 18. High frequency shielding benefits, while reduced somewhat from the $\theta=150^{\circ}$ case, are 2 to $4 \mathrm{~dB}$ and still significant. There is, however, no indication of a low frequency penalty due to jet coalescence at this sideline radiation angle. It is significant to note that while the term shielding is 
used here, the differences observed between the sideline and aft polar angles imply that the individual jets may not be truly shielding in the strictest sense of the term such that individual jets are blocking, or shielding, the radiated acoustic energy from its neighbors. Rather, it is likely that reflection and refraction through the individual jet layers within the plume is the source of the large amount of "shielding" observed in the aft angles. This kind of effect would be smaller at the sideline angles compared to the aft angles, which is what is observed here. Additionally, for hot jet flow where the density gradient between jet columns is more significant than found here, the beneficial effects may increase.

Spectral data analyzed in this way will provide guidance on nozzle geometry improvements resulting in even greater noise reduction for future DEN designs. Variable slot spacing, for instance, may provide a means of realizing the benefits of jet-jet acoustic shielding while eliminating some of the excess low frequency noise due to jet coalescence.

\section{Conclusion}

The data presented here from the DEN designs show a great deal of promise for this technology. Noise reductions up to $20 \mathrm{~dB}$ on a spectral basis and $10 \mathrm{~dB}$ on an overall basis were demonstrated. While the thrust performance penalty is still relatively high, it is approaching levels that could be tolerable in future revolutionary design aircraft systems that integrate the propulsion system into the airframe. It is clear that specific details of a particular DEN design can greatly influence the aeroacoustic properties. Phenomena such as jet-jet mixing, shielding, and coalescence all play a significant role in the resulting noise reduction. Testing and analyses such as those presented here will be required to make distributed exhaust technology viable for an aircraft system. Future system and technology studies at NASA will further explore distributed exhaust nozzles as a candidate for contributing to the $20 \mathrm{~dB}$ perceived noise reduction enterprise goal.

\section{Acknowledgements}

Northrop Grumman performed this work under contract NAS1-02046 funded by the Propulsion Airframe Aeroacoustics subtask of NASA's Quiet Aircraft Technology Program. The authors would like to thank Sang Lee and Meaza Teshome of the NASA Langley Jet Noise Laboratory for their invaluable assistance collecting and processing the acoustic data. The authors would also like to thank Northrop Grumman's Ed Hobart and Gary Carr for their assistance with construction of the stereolithography DEN models and Brian Hallam for timely stress analysis. In addition, Krish Ahuja and Rick Gaeta of Georgia Tech provided helpful insight while comparing the slanted slot DEN results to those reported here. 


\section{References}

1 Kinzie, K. W., Brown, M. C., Schein, D. B., and Solomon, Jr., W. D., "Measurements and Predictions for a Distributed Exhaust Nozzle," AIAA Paper No. 2001-2236 presented at the 7th AIAA / CEAS Aeroacoustics Conference, Maastricht, The Netherlands, May, 2001.

2 Gaeta, R. J., Ahuja, K. K., Schein, D. B., and Solomon, Jr., W. D., "Large Jet-Noise Reductions Through Distributed Exhausts," AIAA Paper No. 20022456 presented at the 8th AIAA / CEAS Aeroacoustics Conference, Breckenridge, Colorado, June, 2002.

3 Ahuja, K.K., Gaeta, R.J., Hellman, B, Schein, D.B., and Solomon, Jr., W.D., "Distributed Exhaust Nozzles for Jet Noise Reduction," GRTI Report A6221/2001-1, Final Report for NASA Contract NAG3-2352, 2001.

4 Shields, F. D. and Bass, H. E., "A Study of Atmospheric Absorption of High Frequency Noise and Application to Fractional Octave Bands of Noise," NASA Contractor Report 2760, 1976

5 Malone, M. B., "Turbulence Model Evaluation for Free Shear Dominated Flows," AIAA Paper No. 962038 presented at the 27th AIAA Fluid Dynamics Conference, New Orleans, Louisiana, June, 1996.

6 Solomon Jr., W. D. and Schein, D. B. "SAJF Distributed Exhaust Nozzle Model Design and Fabrication," NASA Contract No. NAS1-99068 Task 5 Final Technical Report, 2001.

7 Brooks, T. F., Pope, D. S., and Marcolini, M. A., "Airfoil Self-Noise and Prediction," NASA Reference Publication 1218, 1989.

8 Huang, W.J., "Numerical Solution of Aerosound from an Airfoil Using $k-\varepsilon$ Turbulence Model," AIAA 960754, January 1996.

9 Simonich, J. C., Amiet, R. K., and Schlinker, R. H., "Jet Shielding of Jet Noise," NASA Contractor Report 3966, 1986

10 Kantola, R. A., "Shielding Aspects of Heated Twin Jet Noise," AIAA Paper No., 77-1288 presented at the $4^{\text {th }}$ AIAA Aeroacoustics Conference, October, 1977.

11 Wlezien, R. W., Rogers, C. B., Yamamoto, K. J., and Wang, B., "The Effectiveness of Acoustic Shielding for HSCT Nozzle Configurations," AIAA Paper No. 913280 presented at the $9^{\text {th }}$ AIAA Applied Aerodynamics Conference, September, 1991.
Table 1. Expected SPL reduction compared to seven slot case if specified number of slots are flowing; $10 \log (\mathrm{N} / 7)$.

\begin{tabular}{|c|c|}
\hline No. Slots & Delta dB \\
\hline 6 & -0.67 \\
\hline 5 & -1.46 \\
\hline 4 & -2.43 \\
\hline 3 & -3.68 \\
\hline 2 & -5.44 \\
\hline 1 & -8.45 \\
\hline
\end{tabular}




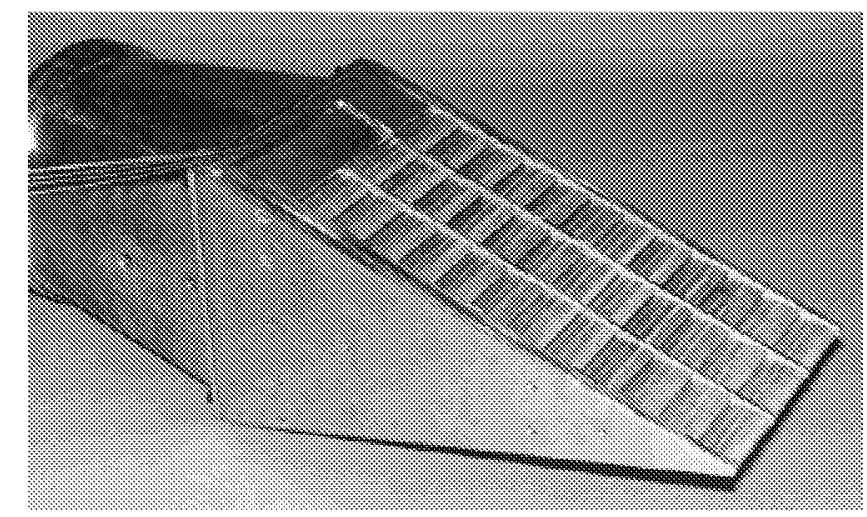

Figure 1. Horizontal slot DEN tested in the NASA LSAWT in 2000.

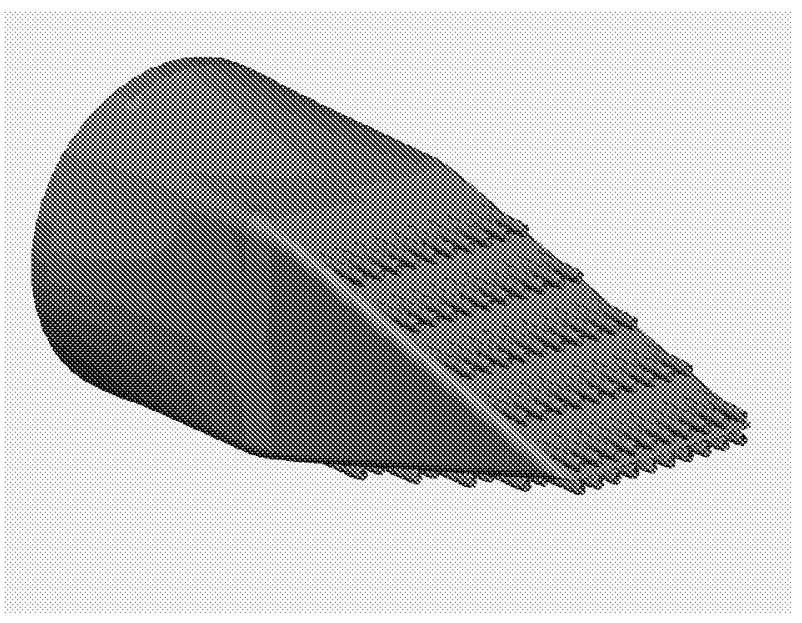

Figure 2. DROPS DEN design tested in NASA SAJF in 2002.

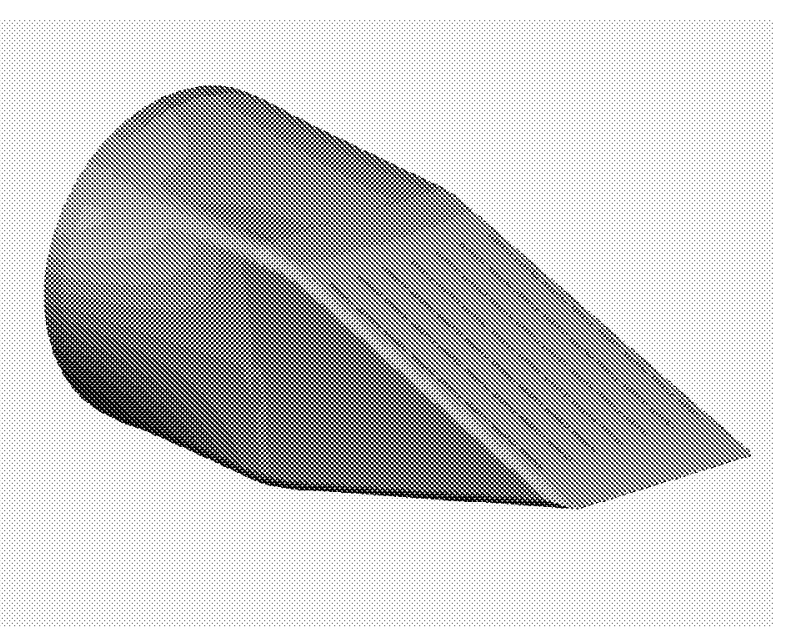

Figure 3. SPS DEN design tested in NASA SAJF in 2002.

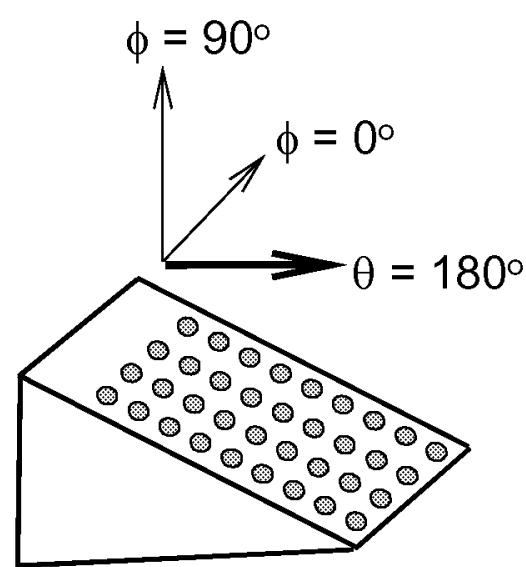

Figure 4. Sketch of nozzle coordinate system and azimuthal orientation planes.

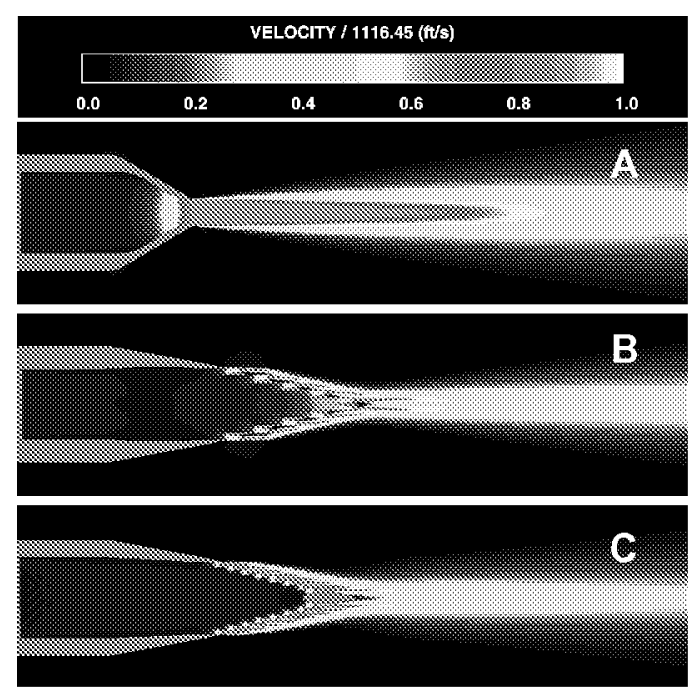

Figure 5. Centerplane velocity magnitude contours for (a) round reference nozzle, (b) DROPS, (c) SPS.

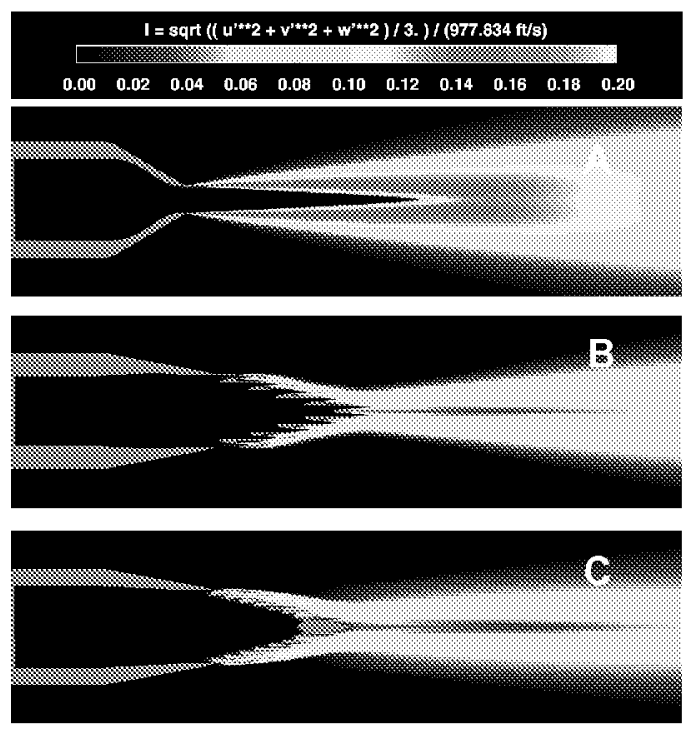

Figure 6. Centerplane turbulence intensity contours for (a) round reference nozzle, (b) DROPS, (c) SPS 


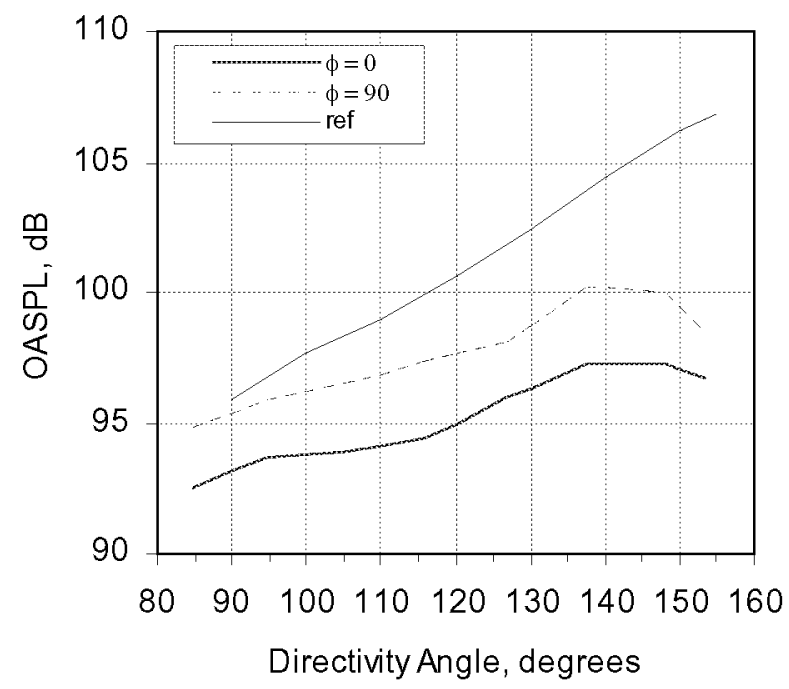

Figure 7. OASPL for DROPS nozzle and reference nozzle at $\mathrm{NPR}=\mathbf{1 . 7 2}$.

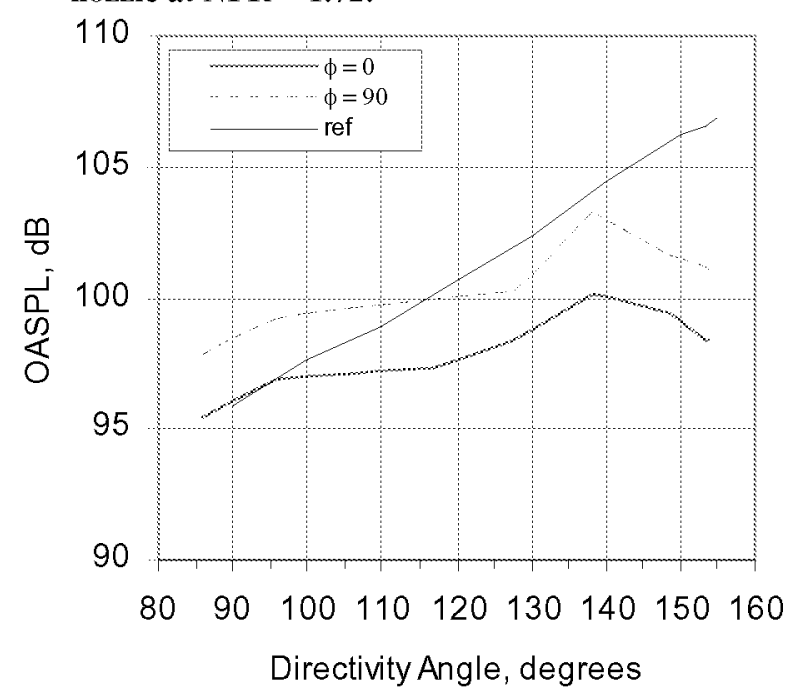

Figure 8. OASPL for SPS nozzle and reference nozzle at $\mathrm{NPR}=1.72$.

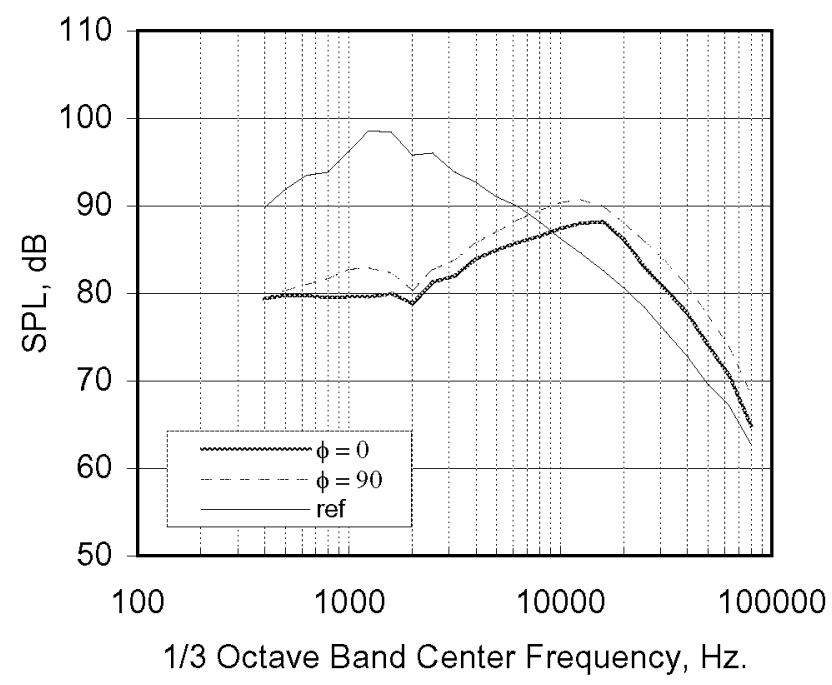

Figure 9. Spectra for DROPS nozzle and reference nozzle at $\mathrm{NPR}=1.72, \theta=150^{\circ}$.

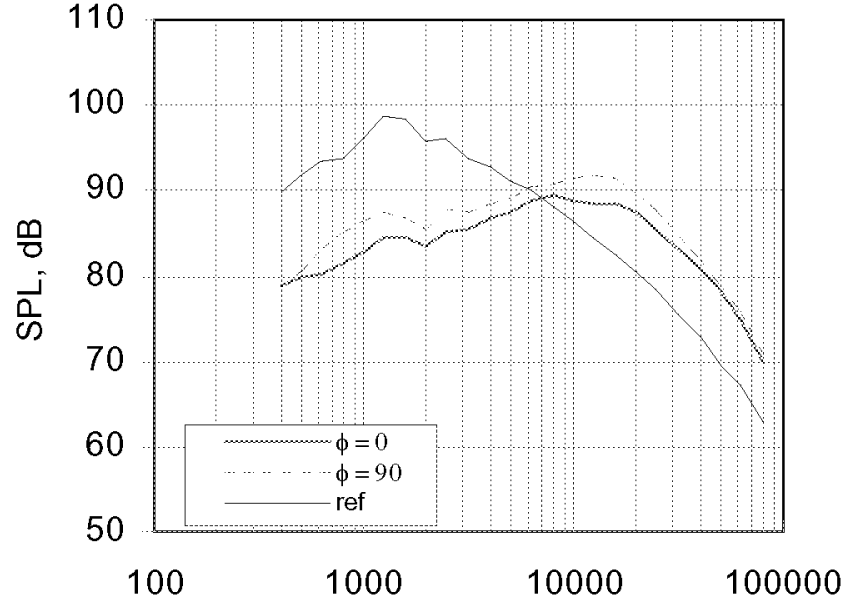

1/3 Octave Band Center Frequency, $\mathrm{Hz}$.

Figure 10. Spectra for SPS nozzle and reference nozzle at $\mathrm{NPR}=1.72, \theta=150^{\circ}$.

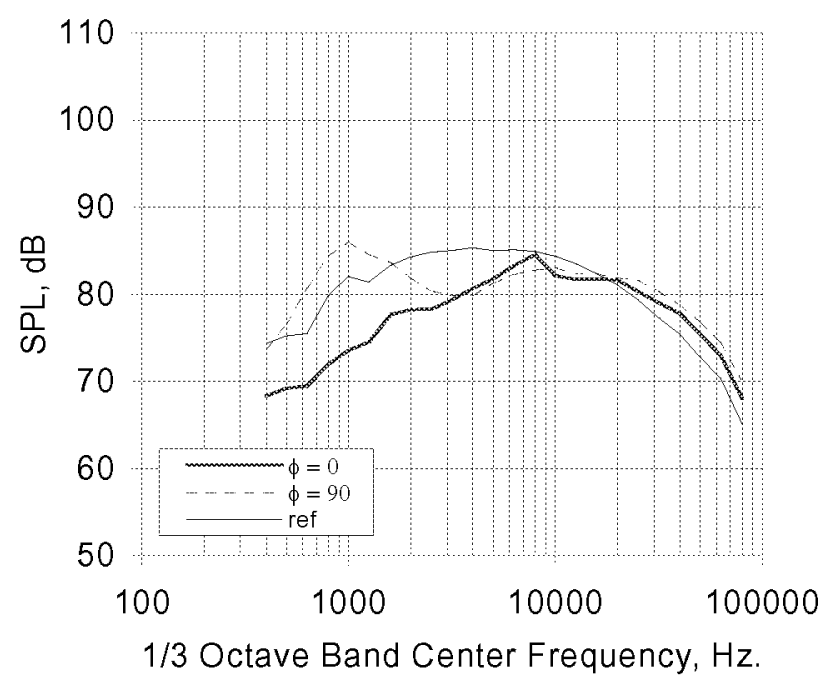

Figure 11. Spectra for DROPS nozzle and reference nozzle at $\mathrm{NPR}=1.72, \theta=90^{\circ}$.

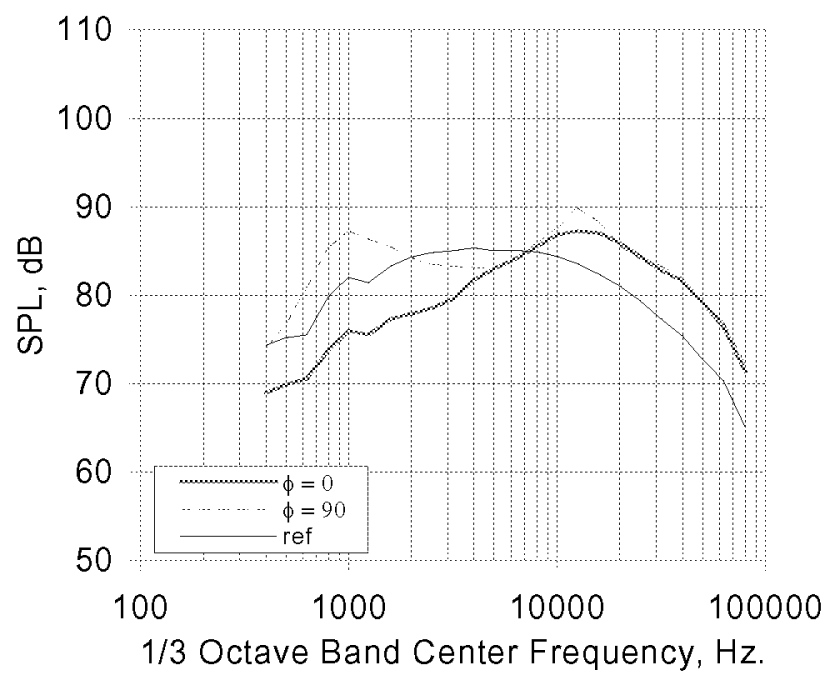

Figure 12. Spectra for SPS nozzle and reference nozzle at $\mathrm{NPR}=1.72, \theta=90^{\circ}$. 


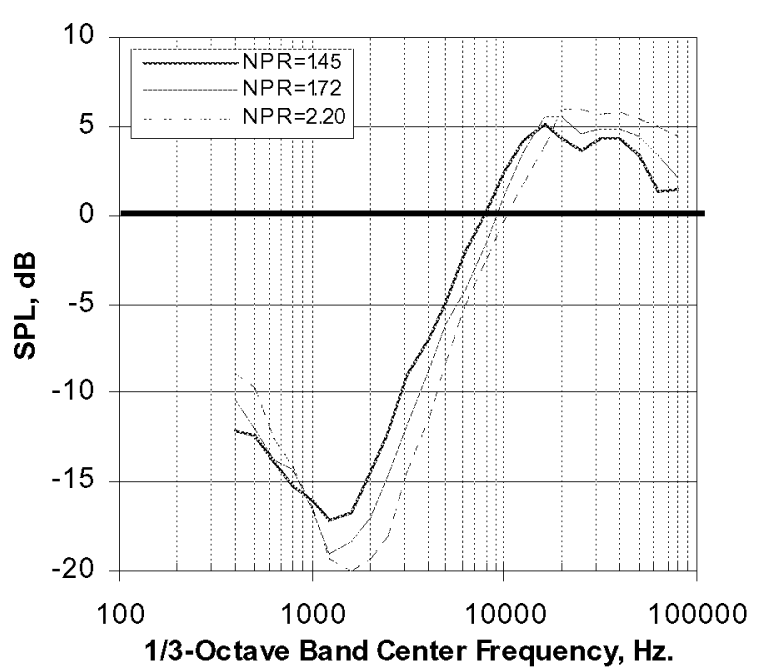

Figure 13. Difference between DROPS nozzle and reference nozzle at $\phi=0^{\circ}, \theta=150^{\circ}$.

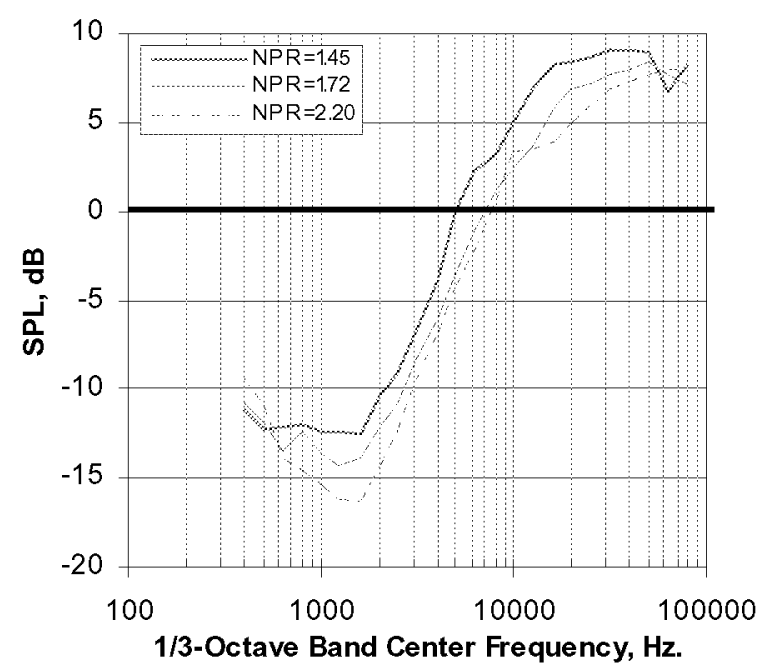

Figure 14. Difference between SPS nozzle and reference nozzle at $\phi=0^{\circ}, \theta=150^{\circ}$.

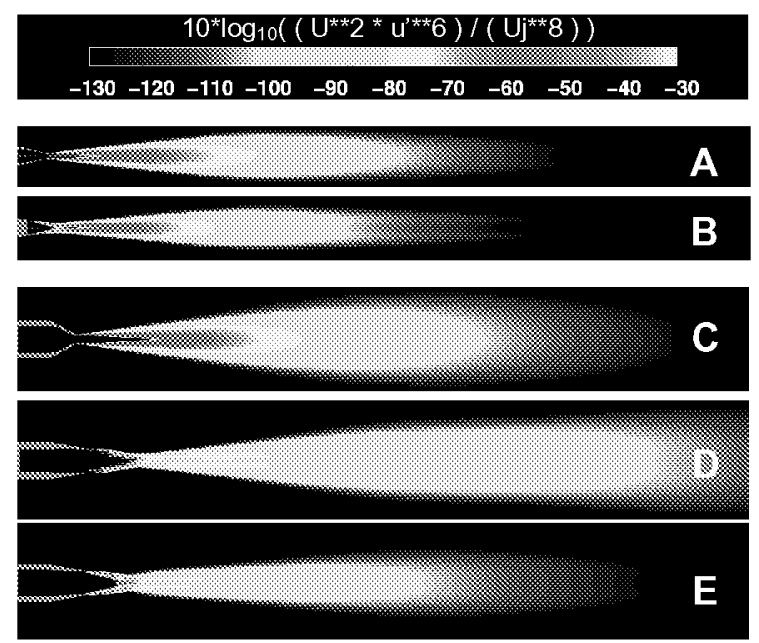

Figure 15. Noise Parameter Comparison, NPR=1.72: A) 2D Ref. (LSAWT), B) Horiz. Slot (LSAWT), C) Ref., D) DROPS, E) SPS

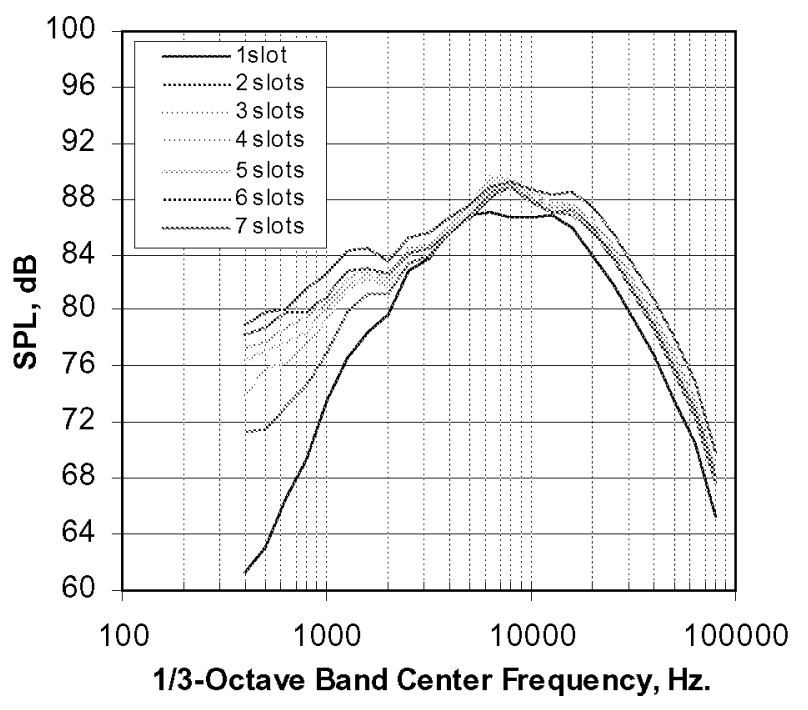

Figure 16. SPS noise shielding data, $\mathrm{NPR}=1.72, \theta=150^{\circ}$.

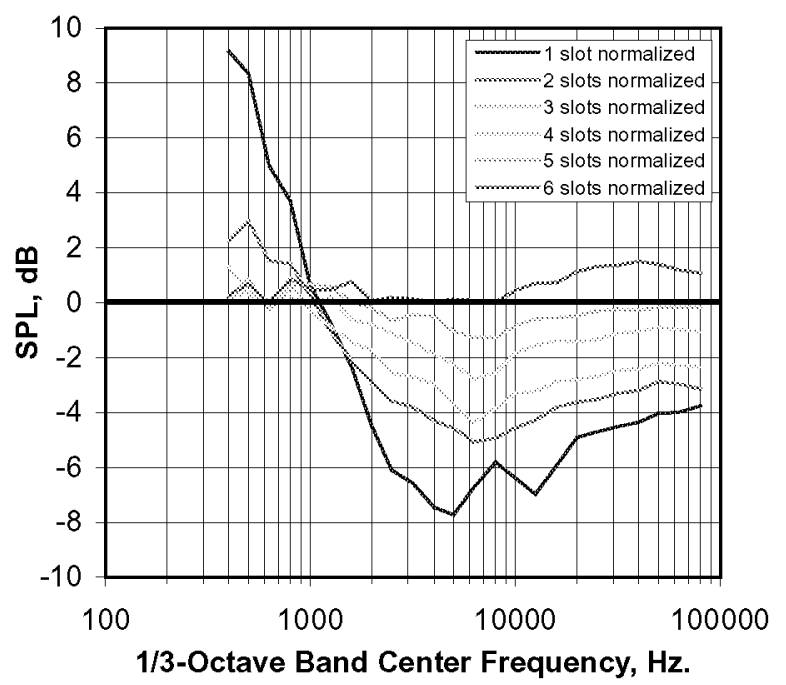

Figure 17. SPS normalized shielding data, $\mathrm{NPR}=1.72, \theta=150^{\circ}$.

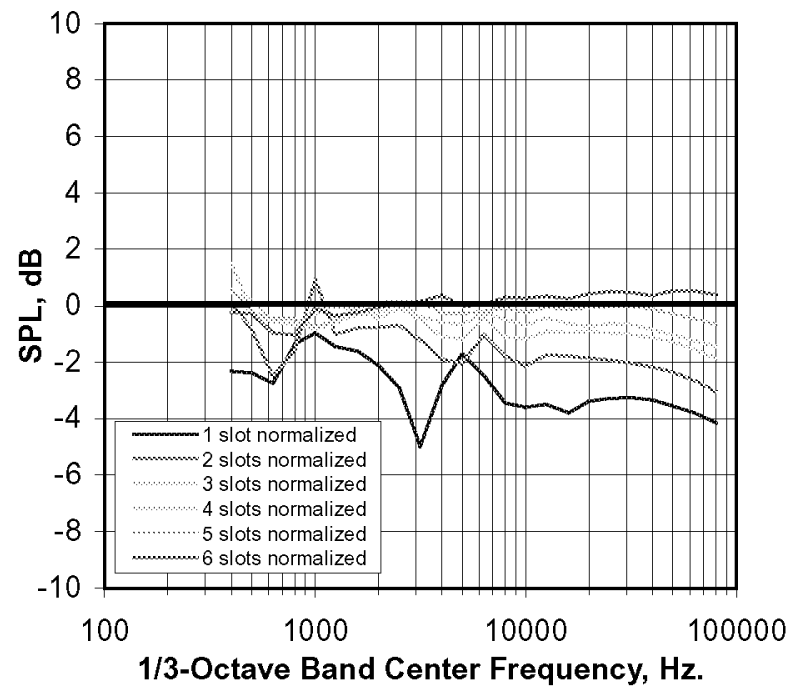

Figure 18. SPS normalized shielding data, NPR=1.72, $\theta=90^{\circ}$. 